\title{
Helen Salisbury: Alone in a darkened room
}

\author{
Helen Salisbury GP \\ Oxford
}

In some general practices, patients seeking help or advice may be seen by a doctor but could equally be given an appointment with a nurse, paramedic, physician's associate, or pharmacist. This may look like a sensible-and cost effective-solution to the current GP workforce crisis.

However, all patients still need a registered GP who ultimately bears responsibility for their care. In this new, multi-professional model, which is most evident in some of the more commercial practice chains, individual GPs can end up with list sizes of over 4000 patients.

When my last patient leaves the surgery at the end of the day, I usually have two or three hours' more work to do. I'll be looking at lab results, signing off prescriptions, reading clinical correspondence, and writing referral letters and medical reports. I admit that I could be more efficient-hours 10, 11, and 12 of my working day tend to be a bit slow-and our systems could be improved, too.

Even with streamlined processes, however, many decisions have to be made, and GPs bear responsibility for any that can't easily be put into a protocol and delegated. Does this marginally low haemoglobin need investigating? That will depend on multiple features of the patient and the history, which may be clear if you know the patient and requested the test but will require more digging if this arose from someone else's consultation. Is it $\mathrm{OK}$ for this patient to continue taking his antidepressants even though he hasn't responded to several requests for a review? This is much easier if you made the original diagnosis.
Taking decisions is tiring. We spend our lives making judgments about what's safe, what's reasonable, and what risks are acceptable. It's even more exhausting when the information we need to make those decisions isn't at our fingertips, in the shape of a patient we already know. In these situations we're likely to become more risk averse, calling patients back for further investigations or appointments, or referring more to secondary care, with a consequent drop in efficient use of resources. There's also a serious risk of burnout in ourselves.

We risk creating a nightmare scenario in which GPs spend most of their time alone in a darkened room, staring at a computer screen. We'd no longer do much of the job we chose and trained to do: consulting with patients we know, diagnosing, treating, caring, and supporting.

I'm appreciative of the skills of other primary care staff, and I look forward to working with an extended team: we may at last be recruiting a pharmacist at my practice who we hope will improve the quality of our prescribing. But, no matter who does the consultations, GPs will still bear overall responsibility for their patients, and this is an increasingly heavy burden as fewer doctors take a share of that load.

Competing interests: See www.bmj.com/about-bmj/freelance-contributors. Provenance and peer review: Commissioned; not externally peer reviewed. Published by the BMJ Publishing Group Limited. For permission to use (where not already granted under a licence) please go to http://group.bmj.com/group/rights-licensing/ permissions 